\title{
Theoretical evaluation of asynchronous AC dielectric nanomotors
}

\author{
Michael Pycraft Hughes \\ School of Engineering, University of Surrey \\ Guildford, Surrey GU2 7XH \\ Tel +44 1483686775 \\ Fax +44 1483689395 \\ Email m.hughes@surrey.ac.uk
}

\begin{abstract}
A design for an electric nanomotor driven by $\mathrm{AC}$ electric fields is presented and evaluated. The motor induces torque by means of a rotating electric field which induces a dipole in the rotor; the interactions between field and dipole are responsible for both the generation of torque and the repulsion of the rotor, which stabilises its position without contact and removes the need for bearings. The applied electric field is generated by square-wave pulses, allowing the direct computer control of the device without any digital-to-analogue requirement; the amount of torque generated can be controlled precisely by varying the number of clock cycles between digital state changes. The technology is scaleable, and a motor consisting of a rotor $1 \mu \mathrm{m}$ long and $100 \mathrm{~nm}$ diameter is shown to be capable of generating approximately $10^{-15} \mathrm{Nm}^{-1}$, equivalent to a bacterial flagellar motor.
\end{abstract}




\section{Introduction}

One of the greatest challenges in engineering at present is that posed by nanotechnology, the development of electromechanical devices on the nanometre scale. These devices, also described as nano-electromechanical systems (NEMS) can be considered to originate from the talk "there's plenty of room at the bottom" by Richard Feynman [1960]. In this, he challenged the audience to devise an electric micromotor measuring $400 \mu \mathrm{m}$ on a side; the challenge was met shortly afterwards. In his subsequent talk, "infinitesimal machinery" [Feynman, 1983] he described a concept for a synchronous dielectric motor; force would be imparted by the stepwise attraction of a dielectric to an electrode, with the potentials on adjacent electrodes being changed so as to "pass" the object from one electrode to another, and so on. A $100 \mu \mathrm{m}$-diameter, rotary motor version of this was later demonstrated by Hagedorn et al [1994] together with asynchronous variants wherein torque was induced by the phenomenon of electrorotation.

AC Electrokinetic techniques such as dielectrophoresis [Jones 1995] and electrorotation [Zimmermann and Neil, 1996] have been utilised for many years for the manipulation and analysis of cellular-scale particles. The phenomenon occurs due to the interaction of induced dipoles with electric fields, and can be used to exhibit a variety of motions including attraction, repulsion and rotation by changing the nature of the dynamic field. In many ways, these forces may be viewed as an electrostatic equivalent to optical tweezers [Ashkin et al. 1986] and optical spanners [Simpson et al. 1997] in that they exert translational and rotational forces on a body due to the interaction between a body and an imposed field gradient. 
Recent advances in semiconductor manufacturing technology have enabled researchers to develop electrodes for manipulating macromolecules with molecular weights as small as 9,000 [Washizu et al. 1994]. This demonstrated the applicability of dielectrophoresis to the manipulation of particle on the nanometre scale, with recent demonstrations including the use of dielectrophoresis to the manipulation of nanowires [Smith et al 2000] and the application of this technique to the construction of nanofibre-based electronic devices [Duan et al 2001]. Carbon nanotubes have also been manipulated using AC dielectrophoretic methods [Yamamoto et al 1998]. Since AC electrokinetic forces can be applied anywhere were current can flow to energise electrodes, they offer a far more practical solution to the actuation of nano-scale motors and gears than the use of laser spanners, such as those described by Srivastava [1997].

In this paper we describe how these techniques might be used to actuate a motor on the nanometre scale, using $\mathrm{AC}$ electrokinetic techniques to drive a rotors down to $1 \mathrm{~nm}$ across. The proposed dielectric nanomotor comprises a dielectric rod (rotor) contained within a chamber consisting of four electrodes which generate a rotating electric field (stator). The electrodes are equidistant from the rotor and run parallel to it. The electrodes can be energised by a square-wave AC potential, phased such that each electrode is $90^{\circ}$ phaseadvanced compared to its predecessor, so that the electric field is observed to rotate. The chamber is filled with a dielectric liquid such as water.

\section{Theory}

Consider a dielectric particle suspended in a spatially non-uniform electric field. The applied field induces a dipole in the particle; the interaction between the charges in each half of the induced dipole and the electric field generates a force. If the electric field is constant on both 
sides of the dipole there is no net force; in the presence of a field gradient, the forces are not equal on the charges at either side of the dipole, and there is a net force which propels the particle. If the particle is more polarisable than the medium around it, the dipole aligns with the field and the force acts up the field gradient towards the region of highest electric field. If the particle is less polarisable than the medium, the dipole aligns against the field and the particle is repelled from regions of high electric field [Jones 1995]. This force was termed dielectrophoresis by Pohl [1979], and the attractive and repulsive cases are referred to as positive and negative dielectrophoresis respectively. The effect is frequency-dependent, and bodies my experience either in different field frequency windows.

If the particle is suspended in a rotating electric field, the induced dipole forms across the particle and rotates in synchrony with the field. However, if the angular velocity of the field is sufficiently large, the time taken for the dipole to form (the relaxation time of the dipole) becomes significant and the dipole lags behind the field. This results in non-zero angle between field and dipole, which induces a torque in the body and causes it to rotate asynchronously with the field; the rotation can be with or against the direction of rotation of the field, depending of whether the lag is less or more than $180^{\circ}$. This effect was termed electrorotation by Arnold and Zimmermann [1982]. These two effects - dielectrophoresis and electrorotation, are generated by the interaction between the in-phase and out-of-phase components of the dipole and the electric field, and as such are different expressions of the same principle [Wang et al 1993]; depending of the nature of the relationship between the electric field and the dipole, a particle may express either or both forces. They are shown schematically in figure 1. 
AC electrokinetic dielectric motors on the micrometre-scale were first presented by Hagedorn et al [1994], who demonstrated electrode arrays manufactured using planar lithography and flat dielectric rotors approximately $100 \mu \mathrm{m}$ across. The model described here extends this by considering the performance of a tall fibre rotor on the nanometre scale. Consider the device shown in figure 2, consisting of an electrode chamber (stator) with four electrodes driven by square-wave potentials in quadrature. At the centre of the chamber is an insulating nanofibre (rotor). The rotor has circular cross-section, identified as producing the greatest torque by Hagedorn et al [1994], and is suspended in water or similar high-permittivity liquid. The electrodes run the length of the fibre. The cross-sectional geometry of the electrode is arbitrary within limits; the truncated prism shape described by Hughes [1998] offers advantages of high torque and large dielectrophoretic forces near the electrode edge.

There is no straightforward expression for determining the induced dipole moment in a rod held perpendicular to an applied electric field. In previous work on the dielectrophoretic forces on rod-shaped virus particles [Morgan and Green 1997], calculations on the dielectrophoretic force exerted on rods have been performed by approximating the rod to a prolate ellipsoid of similar dimensions. However, this approximation can be improved on when the rod is suspended with its axis orthogonal to the plane of the rotating electric field. An improved model can take into consideration the fact that the dipole moment is not concentrated at a point on the shortest circumference of the ellipsoid, but would in fact consist of charge accumulated along the length of the rod. Furthermore, the total dipole moment is due to the total dipole per unit volume, and the volume of a rod and ellipsoid of equal diameter and length will be somewhat different. 
In order to more accurately model of behaviour, we may consider the effect of a twodimensional (circular) dielectric, and then integrate along its length to determine the total dielectric response. In turn, we can approximate this two-dimensional shape as the limiting case (as the short axis tends to zero) of an oblate ellipsoid. This is a special case of dielectrophoretic behaviour, only holding at frequencies above the dielectric dispersion of the rod along its principal axis. At these frequencies, the interfacial charge interaction between rod and field act around the circumference of the body, and the approximation of a series of stacked 2-dimensional circles will hold. Calculations using the ellipsoid model (not shown) indicate that an ellipsoid with major axis length twice that of the minor axis length undergoes dielectric dispersion at approximately half the frequency of the dispersion along the minor axis. This implies that if the rotor is repelled into the centre of the chamber by negative dielectric forces (and has therefore undergone dielectric dispersion), the major axis will have dispersed at a considerably lower frequency than is discussed here and the model presented below is valid, particularly for rotors with much higher axis ratios (of 10:1 or more).

It is known [Hasted 1973] that the electric field induced in an ellipsoid exposed to electric field vector $\mathbf{E}$ applied along axis $a$ is given by

$$
\mathrm{E}_{a}=\frac{\varepsilon_{o}^{*} \mathrm{E}}{\varepsilon_{o}^{*}+A_{a}\left(\varepsilon_{i}^{*}-\varepsilon_{o}^{*}\right)}
$$

Where $\mathrm{A}_{\mathrm{a}}$ is the depolarisation factor along the $a$ axis; and where the complex permittivity is given by

$$
\varepsilon^{*}=\varepsilon-j \frac{\sigma}{\omega}
$$


where $\varepsilon$ is the permittivity, $\sigma$ is the conductivity, $\omega$ the angular frequency of the electric field, and the subscripts o and i refer to the medium (outside the body) and particle (inside the body) respectively. There are three depolarisation factors $A_{a}, A_{b}, A_{c}$ one for each axis. If we consider the case where the field is applied along axis a, then for an infinitesimally flat disk we require the dimension of the ellipsoid to be such that the length across axes $a$ and $b$ are equal, and along axis $c$ tends to zero; we assign these dimensions values a, a and $\delta c$ respectively. If this is the case, it can be shown [Hasted 1973; Jones 1995] that depolarisation factors $A_{a}, A_{b}$ and $A_{c}$ have values of $0.5,0.5$ and approximately zero. The induced polarisation $\mathbf{P}$ per unit volume is given by the expression:

$$
\mathbf{P}_{\mathbf{a}}=\left(\varepsilon_{\mathrm{i}}^{*}-\varepsilon_{\mathrm{o}}^{*}\right) \mathbf{E}_{\mathbf{a}}
$$

Where the induced dipole moment is given by

$$
\mu_{\mathrm{a}}=\frac{4}{3} \pi \mathrm{a}^{2} \delta \mathrm{c} \mathbf{P}_{\mathbf{a}}
$$

From this, the polarisability can be determined thus:

$$
\alpha_{a}=\frac{\mu_{\mathbf{a}}}{\mathbf{E}}
$$

Combining equations (1), (4) and (5) we obtain

$$
\alpha_{\mathrm{a}}=\frac{\frac{4}{3} \pi \mathrm{a}^{2} \delta \mathrm{c}\left(\varepsilon_{\mathrm{i}}^{*}-\varepsilon_{\mathrm{o}}^{*}\right) \varepsilon_{\mathrm{o}}}{\varepsilon_{\mathrm{o}}+\frac{1}{2}\left(\varepsilon_{\mathrm{i}}^{*}-\varepsilon_{\mathrm{o}}^{*}\right)}
$$




$$
\alpha_{\mathrm{a}}=\frac{8}{3} \pi \mathrm{a}^{2} \delta c \varepsilon_{\mathrm{o}} \frac{\left(\varepsilon_{\mathrm{i}}^{*}-\varepsilon_{\mathrm{o}}^{*}\right)}{\left(\varepsilon_{\mathrm{i}}^{*}+\varepsilon_{\mathrm{o}}^{*}\right)}
$$

We can integrate this along axis c to determine the net polarisability of a rod composed of many such circular elements, giving a net polarisability:

$$
\begin{aligned}
& \alpha_{\mathrm{a}}=\int_{0}^{\mathrm{h}} \frac{8}{3} \pi \mathrm{a}^{2} \varepsilon_{\mathrm{o}} \frac{\left(\varepsilon_{\mathrm{i}}^{*}-\varepsilon_{\mathrm{o}}^{*}\right)}{\left(\varepsilon_{\mathrm{i}}^{*}+\varepsilon_{\mathrm{o}}^{*}\right)} \mathrm{dc} \\
& \alpha_{\mathrm{a}}=\frac{8}{3} \pi \mathrm{a}^{2} \varepsilon_{\mathrm{o}} \mathrm{h} \frac{\left(\varepsilon_{\mathrm{i}}^{*}-\varepsilon_{\mathrm{o}}^{*}\right)}{\left(\varepsilon_{\mathrm{i}}^{*}+\varepsilon_{\mathrm{o}}^{*}\right)}
\end{aligned}
$$

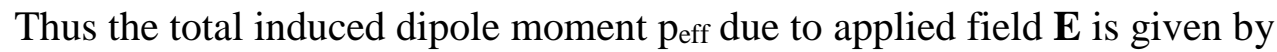

$$
\mathrm{p}_{\text {eff }}=\frac{8}{3} \pi \mathrm{a}^{2} \mathrm{~h} \varepsilon_{\mathrm{o}} \frac{\left(\varepsilon_{\mathrm{i}}^{*}-\varepsilon_{\mathrm{o}}^{*}\right)}{\left(\varepsilon_{\mathrm{i}}^{*}+\varepsilon_{\mathrm{o}}^{*}\right)} \mathbf{E}
$$

It is known that for a dielectric object of induced dipole moment $p_{\text {eff, }}$ the induced electrorotational torque is given by the expression

$$
\Gamma=\frac{1}{2}\left[p_{\text {eff }} x \mathbf{E}\right]
$$

Assuming $\mathrm{E}$ is of the form

$$
\mathbf{E}=\mathrm{E}_{\mathrm{o}}(\hat{\mathrm{x}}+\mathrm{j} \hat{\mathrm{y}})
$$


where $E_{o}$ is the peak field, $\hat{x}$ and $\hat{y}$ are orthogonal vectors and $j=\sqrt{-1}$, then the net rotational torque along the rod is given by the expression

$$
\Gamma=\frac{8}{3} \pi \mathrm{a}^{2} \mathrm{~h} \varepsilon_{\mathrm{o}} \operatorname{Im}\left[\frac{\left(\varepsilon_{\mathrm{i}}^{*}-\varepsilon_{\mathrm{o}}^{*}\right)}{\left(\varepsilon_{\mathrm{i}}^{*}+\varepsilon_{\mathrm{o}}^{*}\right)}\right] \mathbf{E}^{2}
$$

Similarly, the particle experiences a dielectrophoretic force given by the expression

$$
\begin{aligned}
& \mathbf{F}=\mathrm{p}_{\mathrm{eff}} \cdot \nabla \mathbf{E} \\
& \mathbf{F}=\frac{8}{3} \pi \mathrm{a}^{2} \mathrm{~h} \varepsilon_{\mathrm{o}} \operatorname{Re}\left[\frac{\left(\varepsilon_{\mathrm{i}}^{*}-\varepsilon_{\mathrm{o}}^{*}\right)}{\left(\varepsilon_{\mathrm{i}}^{*}+\varepsilon_{\mathrm{o}}^{*}\right)}\right] \nabla \mathbf{E}^{2}
\end{aligned}
$$

In both cases, the frequency response of the system is dictated by the relationship

$$
\frac{\left(\varepsilon_{\mathrm{i}}^{*}-\varepsilon_{\mathrm{o}}^{*}\right)}{\left(\varepsilon_{\mathrm{i}}^{*}+\varepsilon_{\mathrm{o}}^{*}\right)}
$$

It was demonstrated by Hagedorn et al (1994) that negative (repulsive) dielectrophoresis can be used to maintain the position of the rotor at the centre of the electrode array, at an electric field null. This confers a great benefit to the motor in that the rotor is held in place without any physical contact, and therefore experiences no torque except the frictional force between the suspending medium and the rotor as it rotates. The presence of this suspending medium limits the applications to which the motor can be put; nevertheless, it is essential for negative dielectrophoresis to take place that the rotor be less polarisable than the material between the rotor and stator - a condition that cannot take place in a vacuum. A possibility which might 
be explored would be to enclose the rotor in a low-pressure plasma, but this is not discussed here and remains for future work.

\section{Ultimate limits of motor performance}

We can use equation (13) to determine the ultimate performance limits of a dielectric nanomotor. If we examine the expression, we find that for a given frequency (i.e. constant values of complex permittivity in particle and medium) the variables on which the torque is dependent are:

$$
\Gamma \propto \mathbf{a}^{2} h \mathbf{E}^{2}
$$

If we consider the rotation chamber as enclosing a central region across which the electric field is approximately uniform and equal to the potential on opposing electrodes divided by the distance between those electrodes [Hughes 1998], then we can replace the $\mathbf{E}$ term thus:

$$
\mathbf{E}=\frac{\mathrm{V}}{2 \mathrm{ka}}
$$

where $\mathrm{k}$ is the ratio between the diameter of the rotor and the distance between opposing electrodes. Substituting equation (18) into equation (17) produces:

$$
\Gamma \propto \frac{\mathrm{h} \mathbf{V}^{2}}{\mathrm{k}^{2}}
$$


Interestingly, this indicates that the torque generated by the motor is not related to the diameter of either the rotor or stator; only on the length of the rotor and the ratio of rotor and stator size.

However, there is a natural limit imposed on this system; the applied electric field may not exceed the dielectric breakdown voltage of water (assuming water to be the medium in which the rotor is suspended). In essence, this imposes a maximum value of $\mathbf{E}$ of approximately $20 \mathrm{MVm}^{-1}$. If this is considered to be a constant value for calculating the upper limit of motor performance, then at the maximum electric field limit the governing equations can be written as:

$$
\left.\begin{array}{l}
\Gamma \propto \mathrm{a}^{2} \mathrm{~h} \\
\mathbf{V} \propto \mathrm{ka}
\end{array}\right\}
$$

indicating that the key factor in torque generation is the volume of the rotor, and the value of the applied potential is proportional to the diameter of the stator chamber. However, in nanomotor design we can assume these are in conflict - it is preferable to have a large torque, small rotor and low driving voltage. This is best reconciled by defining a supply voltage, which defines a value of $\mathrm{a}$, and then use the variation in $\mathrm{h}$ to adjust maximum motor torque as required. The value of $\mathrm{k}$ should be as near unity as possible - i.e. the rotor should fill as much of the chamber as possible - but work by Hughes [1998] indicates that the maximum ratio on region where torque is relatively stable is about $1.5: 1$. 
If we consider a motor with fixed length of $1 \mu \mathrm{m}$ and $\mathrm{k}=1.5$, then we can calculate the variation in torque for a range of rotor widths and applied potentials. Figure 3 shows the response of a hypothetical motor in water $\left(\varepsilon_{\mathrm{r}}=78\right)$. Torque was calculated using equation (13) with the constraints of equations (19) and (20), and a maximum permitted electric field strength of $20 \mathrm{MVm}^{-1}$ was applied. The value of the imaginary part of the complex permittivity relationship was assumed to have a value of 1 . No data are entered for combinations of values of $\mathrm{E}$ which exceed this maximum. As can be seen, torques exceeding $0.1 \mathrm{pNm}$ can be generated by rotor radii with radii greater than $300 \mathrm{~nm}$, but for rotors smaller than this, the peak torque is limited. The absent region in the graph indicates the "forbidden values" where the voltage must be limited according to equation (20). The variation defined by the conditions in equation (20) exist along the diagonal edge of the graph, and indicate the maximum torque for a given value of rotor dimension regardless of applied potential. The maximum torque which can be generated by a $10 \mathrm{~nm}$-radius rotor is $2.3 \times 10^{-16} \mathrm{Nm}$; for a $1 \mathrm{~nm}$ radius rotor the value is $2.3 \times 10^{-18} \mathrm{Nm}$. This torque would require a stator chamber $3 \mathrm{~nm}$ wide and driven with an RMS voltage of $60 \mathrm{mV}$.

\section{Frequency variation of torque and stabilising force}

Having described the variation in torque as a function of dimension and applied potential, we now turn to the relationship between torque and the frequency of the applied electric field. The torque expression in equation (13) shows a strong correlation between the torque generated and the complex permittivities of the particle and medium - both of which are frequency dependent. Furthermore, the stabilising dielectrophoretic force is also frequency 
dependent, and will only repel the rotor in a certain frequency range. In order to demonstrate this, we will consider a numerical example.

Consider a device shown in figure 2, consisting of an electrode chamber (stator) $150 \mathrm{~nm}$ in diameter, with the electrodes are driven by a $1 \mathrm{~V}_{\text {rms }}$ potential. At the centre of the chamber is an insulating nanofibre (rotor) $100 \mathrm{~nm}$ diameter, and $1 \mu \mathrm{m}$ long and having internal relative permittivity 2.55 and net effective conductivity (including both surface and internal components, as discussed in Hughes et al [1999]) $10 \mathrm{mSm}^{-1}$. The rotor is suspended in water (conductivity $1 \mathrm{mSm}^{-1}$, relative permittivity 78). A numerical simulation of this motor was performed using the ANSYS ${ }^{\mathrm{TM}}$ finite element software package, using the motor dimensions and electrical properties outlined above. The simulation indicated that the electric field strength across the volume occupied by the rotor was approximately $1.5 \times 10^{7} \mathrm{Vm}^{-1}$; the electric field gradient in the region between the edge of the rotor and the electrodes was $1.33 \times 10^{21} \mathrm{~V}^{2} \mathrm{~m}^{-3}$. The frequency-dependent torque and stabilising force of the motor response of this motor, according to equations (13) and (15), are shown in figure 4. As can be seen, the peak torque generated by the motor is $1.3 \times 10^{-15} \mathrm{Nm}$ in a frequency window between 2$3 \mathrm{MHz}$. The peak force propelling the rotor to the centre of the motor is approximately $18 \mathrm{nN}$ at frequencies above approximately $6 \mathrm{MHz}$. Combining these, we suggest the optimum condition for the motor is to generate torque between 0.1 and $1 \times 10^{-15} \mathrm{Nm}$ by varying the applied frequency between $5 \mathrm{Mhz}$ and $50 \mathrm{MHz}$ (indicated by the shaded region in figure 4 ). Control over torque below $0.1 \times 10^{-15} \mathrm{Nm}$ can of course be achieved by the application of higher frequencies. Under these operating conditions, the stabilising dielectrophoretic force does not drop below $10 \mathrm{nN}$. 
This can be compared to the rotary motor used by flagellate bacteria to provide propulsion [DeRosier 1998]. These rotary motors are typically 100nm long and 50nm in diameter, and drive the flagellum by means of a "stepper motor" process driven by 2000 torque-generating units, each of which is powered by protons travelling across a $100 \mathrm{mV}$ potential gradient, in a process which is not yet understood. The total torque produced by a bacterial motor is approximately $1.5 \times 10^{-15} \mathrm{Nm}$, comparable with the dielectrophoretic motor described above. The biological nanomotor is somewhat smaller than the electromechanical device described here, but has limited control over torque and which requires a proton gradient across the motor. The electromechanical variant is considerably less efficient in terms of required voltage for torque generation, and is somewhat larger, but gains over the biological variant in that it can be directly powered and controlled by a computer, can be fabricated by conventional ULSI (ultra-large scale integration) and allows precision control over the amount of torque generated.

The low-frequency region in which the rotor experiences positive (attractive) dielectrophoresis also has an application in the construction of the device. Whilst the electrode chamber can be constructed using planar methods like e-beam lithography, the rotor must be placed in the chamber after construction. By applying a solution containing a number of rotor filaments to the unfilled chamber, positive dielectrophoresis can be used to attract a rotor towards, and ultimately into, the chamber. Once a rotor occupies the chamber the frequency can be changed to bring about a repulsive force, so that no further filaments enter. A similar practice has been demonstrated for attracting latex beads into field cages, where it has been demonstrated to be selective enough to trap single $93 \mathrm{~nm}$-diamter latex spheres [Hughes and Morgan 1998]. This is also interesting as the construction features elements of both "top-down" (conventional lithography) and "bottom-up" (self assembly) 
construction techniques outlined by Drexler [1992]. It should also be noted that the ability to switch to an attractive mode is useful in allowing the rotor to be "parked" or locked in position. Braking is not an issue due to the much greater effect of viscous drag on particles of this size.

Due to the nature of the nanomotor presented here requiring that the rotor be submersed in some dielectric liquid in order to function optimally, this places restrictions on the range of applications to which the motor might be put. We suggest that the most appropriate applications will be in the movement of liquid around a sample, or perhaps most appropriately given its resemblance to the bacterial rotary motor, in the locomotion of micrometre-scale devices around the body for clinical applications as described by Feynman [1983]. Coupling between the end of the rotor and subsequent components could be achieved with chemical coupling, for example by using antibody-antigen conjugation, with an appropriate antibody attached to the end of the rotor and a secondary antibody attached to a subsequent component such as a propeller, or with avidin-biotin coupling.

\section{Digital electronic control of torque generation}

It has been demonstrated by Gimsa et al. [1989] that square-wave, rather than sinusoidal, signals can be used effectively in the activation of electrodes for electrorotation; the effect is equivalent to the summation of torques induced by the fundamental and higher frequency partials. The torque is largely unaffected as only the lowest components above the fundamental are responsible for generating torque (as the torque generated diminishes rapidly at applied field frequencies a decade above the resonant peak), and any variation from the torque-frequency characteristic shown in figure 4 can be accounted for via a frequency-torque lookup table. The principal advantage of the application of square wave signals over 
sinusoids is that it allows direct control of the generation of electric signals by a computer. Variation in frequency can be achieved by altering the number of system clock cycles in the "electrode on" and "electrode off" states; that is, by varying the length of a series of 1's followed by an equal number of 0 's. Opposing phases $\left(0^{\circ} / 180^{\circ}\right.$ and $\left.90^{\circ} / 270^{\circ}\right)$ can be achieved with NOT gates. If a further NOT gate is applied to the signals powering one set of opposing electrodes, then the direction of rotation is reversed. The example of torque generation presented above is equivalent to a controlling signal with $\mathrm{V}_{\mathrm{OFF}}=0 \mathrm{~V}$ and $\mathrm{V}_{\mathrm{ON}}=2.8 \mathrm{~V}$, similar to the core voltage of modern microprocessors.

\section{Conclusions}

A design for an electric nanomotor driven by $\mathrm{AC}$ electric fields has been presented. The motor induces torque by induction of $\mathrm{AC}$ electrokinetic effects (dielectrophoresis and electrorotation) in the rotor; providing both torque and contactless position stabilisation. The torque generated in similar to that generated by bacterial flagellar motors, and consequently it is suggested that the motor may be applicable to the propulsion of liquid both for pumping applications, and in the propulsion of micrometre-scale electronic devices in aqueous environments.

\section{Acknowledgements}

The author thanks Mr Z. Ghazzawi for help with the ANSYS simulation. 


\section{References}

Arnold WM, Zimmermann U (1982) Z. Naturforsch. Vol. 37c, pages 908-915. Rotatingfield-induced rotation and measurement of the mambrane capacitance of single mesophyll cells of Avena sativa.

Ashkin A, Dziedzic JM, Bjorkholm JE, Chu S (1986) Optics letters Vol. 11 pages 288-290. Observation of a single-beam gradient force optical trap for dielectric particles.

DeRosier DJ (1998) Cell. Vol. 93 pages 17-20. The turn of the screw: the bacterial flagellar motor

Duan X, Huang Y, Cui Y, Wang J, Lieber CM (2001) Nature. Vol. 409 pages 66-69. Indium phosphide nanowires as building blocks for nanoscale electronic and optoelectronic devices.

Drexler, KE (1992) Nanosystems; molecular machinery, manufacturing and computation. (New York: Wiley)

Feynman R (1960) reprinted in J. Microelectromechanical systems (1992) Vol. 1 pages 6066. There's plenty of room at the bottom.

Feynman R (1983) reprinted in J. Micromechanical Systems (1993) Vol. 2 pages 4-14. Infinitesimal machinery.

Gimsa J, Pritzen C, Donath E (1989) Studia Biophysica Vol. 130 pages 123-131. Characterisation of virus-red-cell interaction by electrorotation.

Hagedorn R, Fuhr G, Müller T, Schnelle T, Schnakenberg U, Wagner B (1994) J. Electrostatics Vol. 33 pages 159-185. Design of asynchronous dielectric micromotors. Hasted JB (1973) Aqueous Dielectrics (London: Chapman and Hall)

Hölzel R (1994) IEEE Trans. Inst. Meas. Vol. 42 pages 758-760. A simple wide-band sinewave quadrature oscillator. 
Hughes MP, Morgan H, Flynn MF (1999) Journal of Colloid and Interface Science Vol. 220 pages 454-457. Surface Conductance in the Diffuse Double-Layer Observed by Dielectrophoresis of Latex Nanospheres.

Hughes MP (1998) Physics in Medicine and Biology Vol. 43 pages 3639-3648. Computeraided analyses of conditions for optimising practical electrorotation.

Jones, TB (1995) Electromechanics of Particles (Cambridge, MA: Massachusetts Institute of Technology)

Morgan H, Green NG (1997) Journal of Electrostatics Vol. 42 pages 279-293. Dielectrophoretic manipulation of rod-shaped viral particles.

Pohl HA (1978) Dielectrophoresis (Cambridge: Cambridge University Press)

Simpson NB, Dholakia K, Allen L, Padgett MJ (1997) Optics Letters Vol. 22 pages 52-54. Mechanical equivalence of spin and orbital angular momentum of light: an optical spanner.

Smith PA, Nordquist CD, Jackson TN, Mayer TS, Martin BR, Mbindyo J, Mallouk TE (2000) App. Phys. Lett. Vol. 77 pages 1399-1401. Electric-field assisted assembly and alignment of metallic nanowires.

Srivastava D (1997) Nanotechnology Vol. 8 pages 186-192. A phenomenological model of the rotation dynamics of carbon nanotube gears with laser electric fields.

Wang X-B Huang Y, Holzel R, Burt JPH and Pethig R (1993) J. Phys. D: Appl. Phys. Vol. 26 pages 312-322. Theoretical and experimental investigations of the interdependence of the dielectric, dielectrophoretic and electrorotational behaviour of colloidal particles.

Washizu M, Suzuki S, Kurosawa O, Nishizaka T, Shinohara T (1994) IEEE Trans. Ind. Appl. Vol. 30 pages 835-842. Molecular dielectrophoresis of biopolymers.

Yamamoto K, Akita S, Nakayama Y (1998) J. Phys. D: Appl. Phys. Vol. 31 pages L34-L36. Orientation and purification of carbon nanotubes using ac electrophoresis. 
Zimmermann U, Neil GA (1996) Electromanipulation of cells. (CRC press: Boca Raton) 


\section{Figure legends}
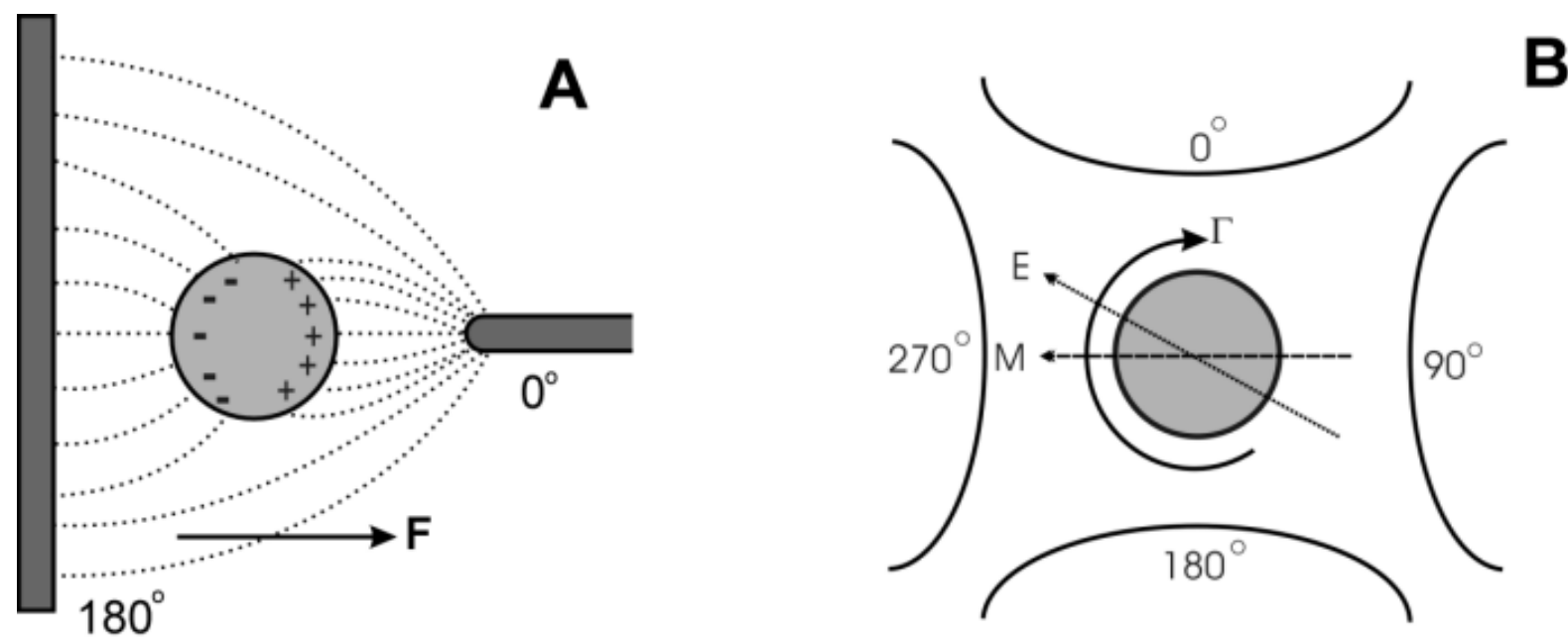

1. Diagrams explaining the operation of the two key $\mathrm{AC}$ electrokinetic forces. (a)

Dielectrophoresis occurs when a polarisable particle is suspended in a non-uniform electric field, due to the interaction between the dipole and the local electric field. (b) Electrorotation occurs when the field is rotating, so that there is an angular displacement between the dipole and electric field which in turn generates a torque.

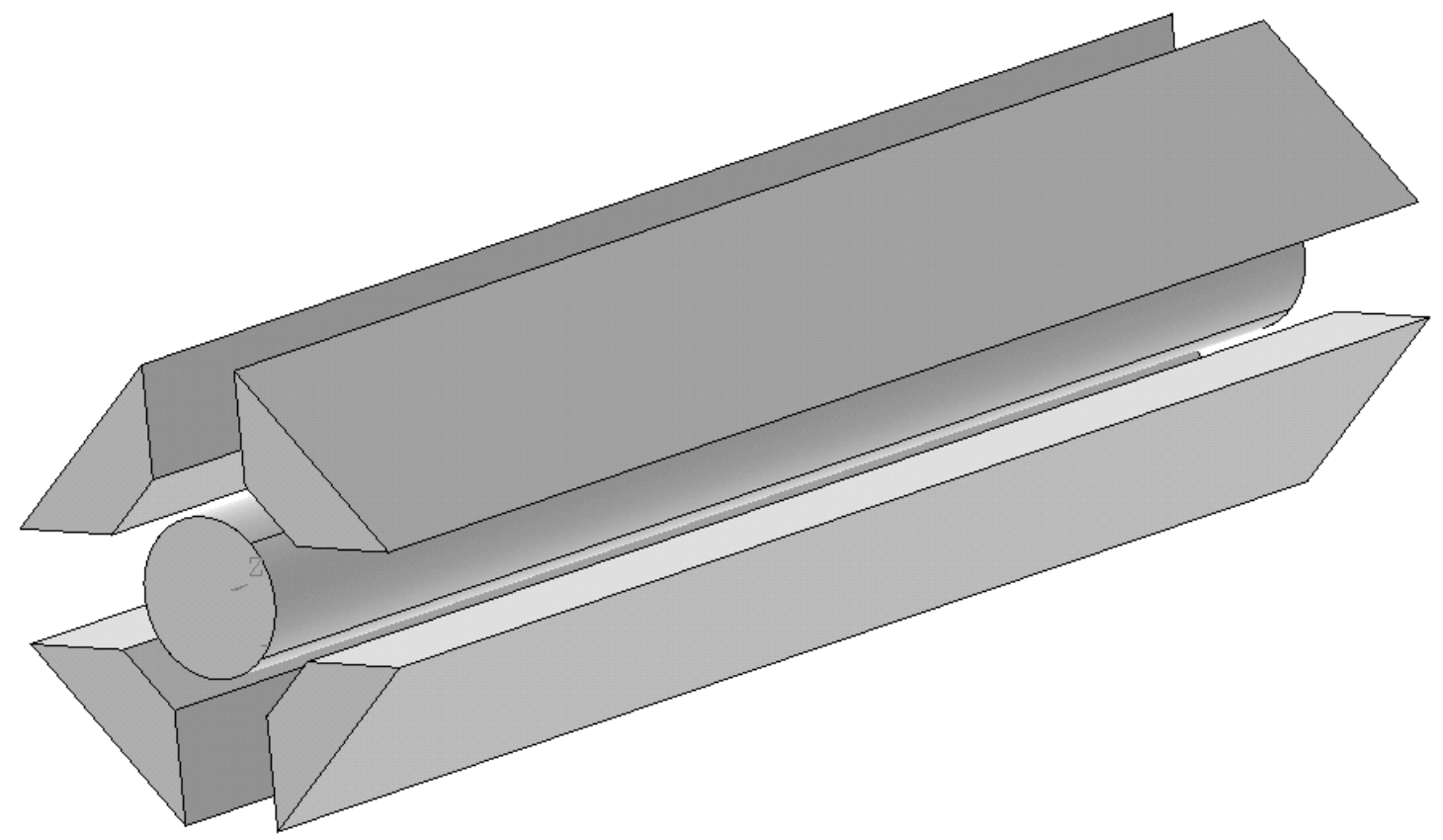


2 A schematic of the nanomotor. Four truncated-prism electrodes (the stator) surround a central nanofibre (rotor). The electrodes are energised by potentials in quadrature, such that the phase of the wave on each electrode leads its clockwise neighbour by $90^{\circ}$. 


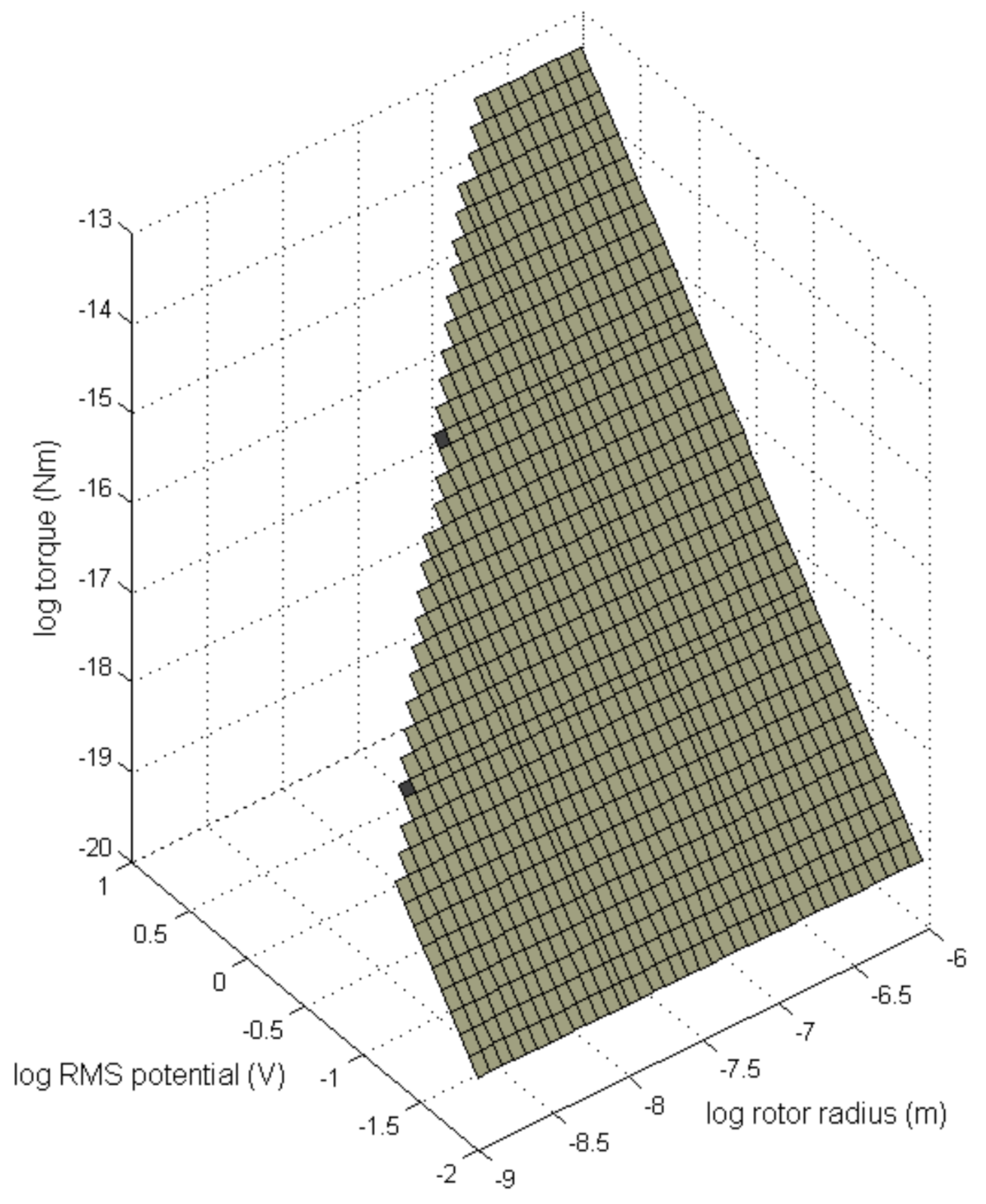

3. A graph of the variation in torque developed by a nanomotor $1 \mu \mathrm{m}$ long, with rotor radius a and applied RMS potential $\mathrm{V}$, in a stator chamber 1.5 times wider than the rotor. As can be seen, the torque generated increases with applied potential and is 
independent of rotor radius; however, the limitation that the electric field in the chamber cannot exceed the electrical breakdown conditions of water restricts the maximum torque which can be developed by small rotors. Combinations of values which break this condition are not illustrated.
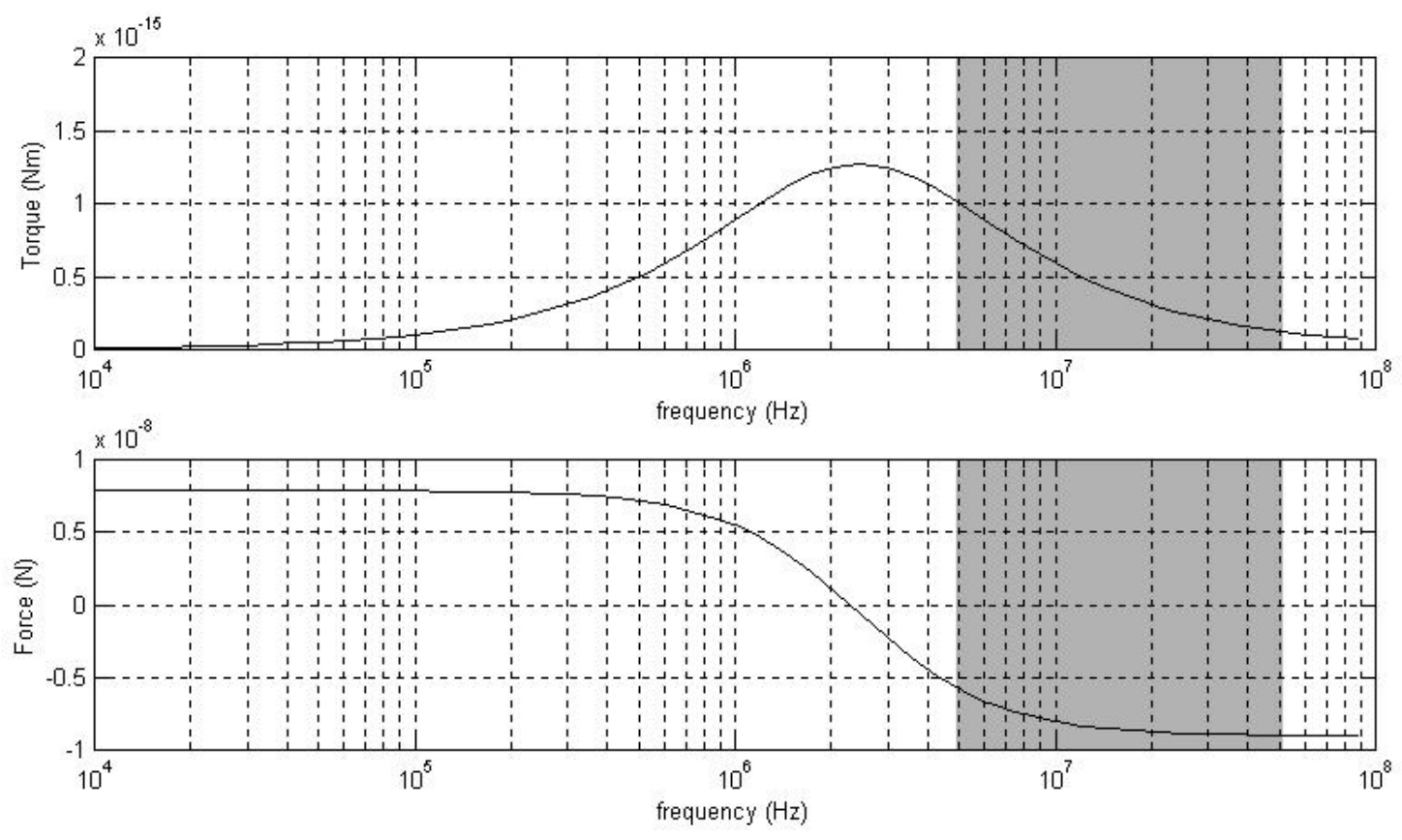

4. The forces generated in a $100 \mathrm{~nm}$-diameter, $1 \mu \mathrm{m}$-long rotor under the conditions described in the text. The top figure illustrates the frequency-dependent torque on the rotor; the lower figure indicates the frequency-dependent dielectrophoretic force between the rotor and the electrodes. At frequencies above $2.3 \mathrm{MHz}$, the rotor experiences negative dielectrophoretic force and will be electrostatically suspended in the centre of the chamber, removing the need for bearings. The shaded band corresponds to the frequencies of optimum performance described in the text. 